\title{
Probing the formation of stable tertiary structure in a model mini-protein at atomic resolution: Determinants of stability of a helical hairpin
}

Alexey Neumoin, Jiri Mares, Mirjam Lerch-Bader, Reto Bader and Oliver Zerbe

\section{Supporting information}

Figure S1: Representative structures of Ala14-pPYY (left), pPYY (middle) and bPP (right).
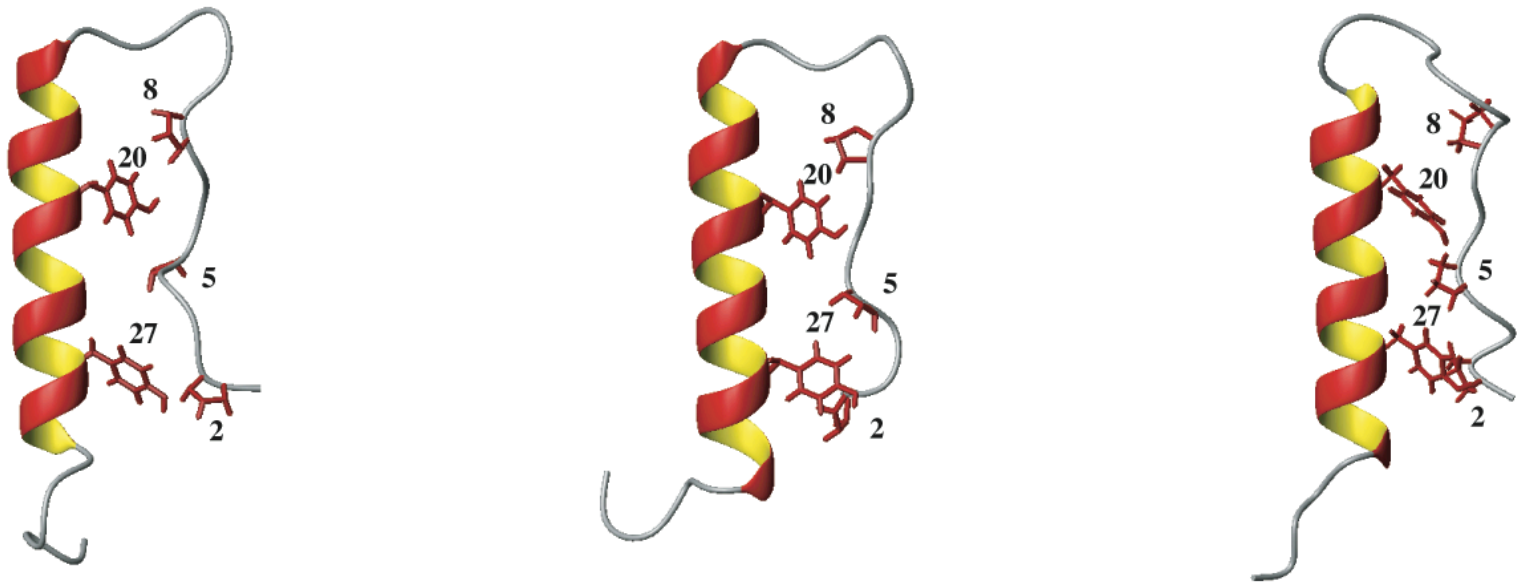

Figure S2: Structure of Tyr7-PYY as determined at $\mathrm{pH} 4.1,301 \mathrm{~K}$ : Backbone presentation of the superposition of single 20 lowest energy conformers (left), and single conformer (middle) with sidechains of Pro and Tyr residues depicted (right).
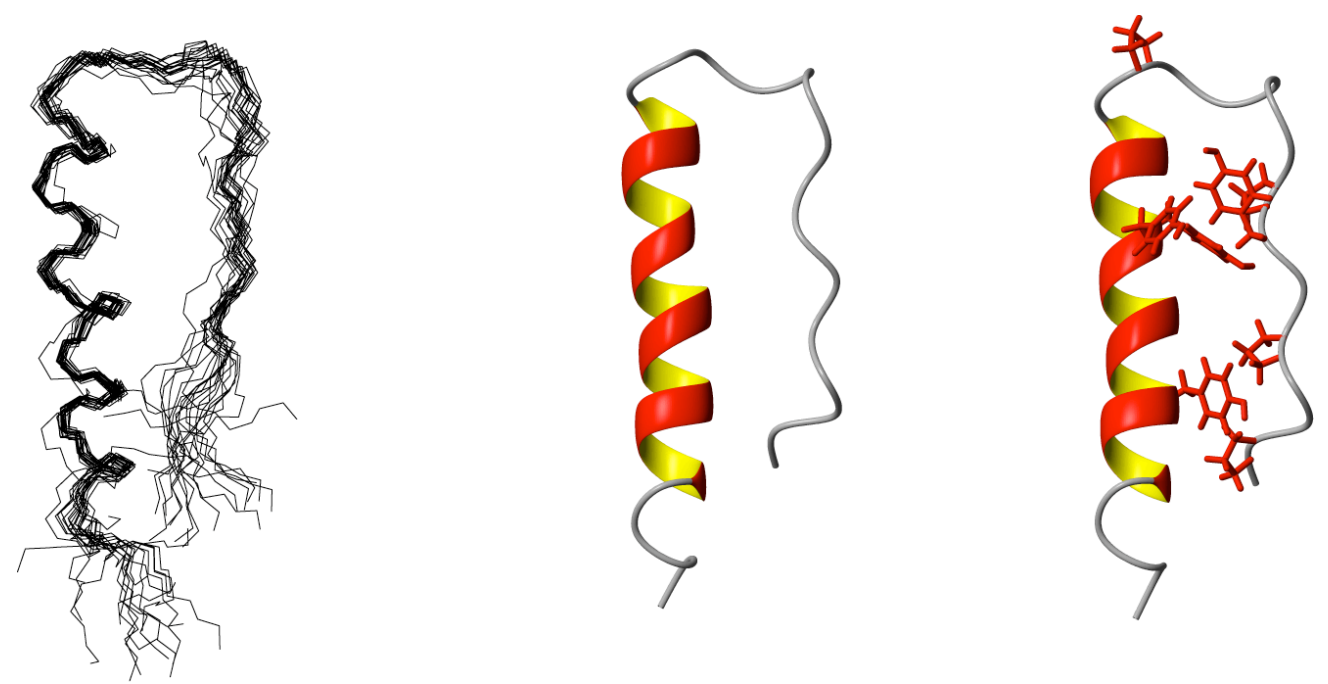
Figure S3: ${ }^{15} \mathrm{~N}\left\{{ }^{1} \mathrm{H}\right\}-\mathrm{NOE}$ of PYY, Ala2-PYY, Ala5-PYY, Ala8-PYY and Ala2,5,8,14-PYY at pH 6.0 in $20 \mathrm{mM}$ MES buffer, 300mM DPC using the same color coding and symbol as in Fig. 2.

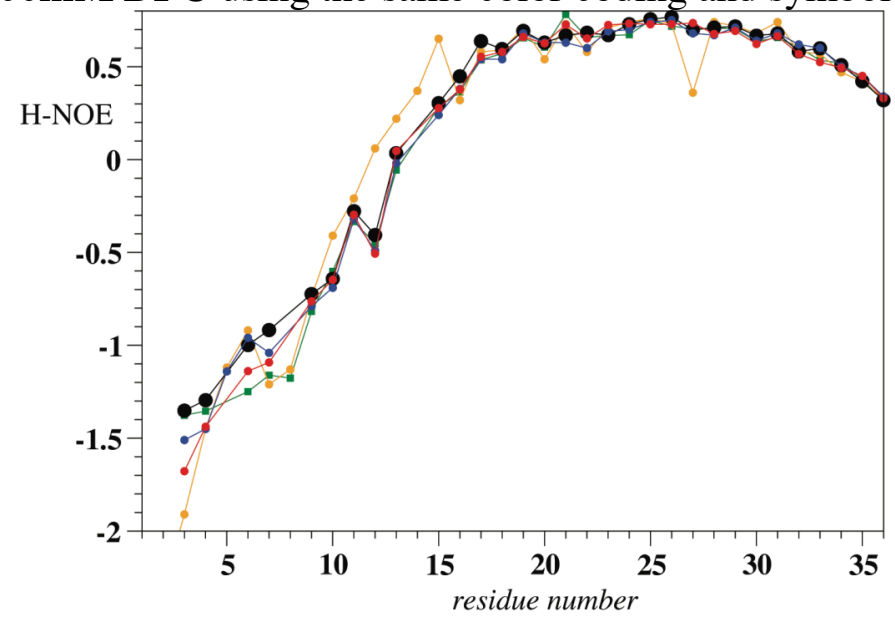

Solvent mixture (methanol-water) experiments for Tyr7-PYY:

Mixtures of water and methanol may be particularly useful to study helical hairpin formation in PYY. We have followed the amide proton chemical shifts $v s$. the methanol content. The data for selected residues of Tyr7-PYY, for which the transition between non-back-folded and back-folded species occurs at a larger methanol-to-water ratio compared to PYY and hence is better visible. i) Chemical shifts of residues 3,4,6,7,9,10, 12 17, 18 (23) and 24 of Tyr7-PYY, which are all part of the back-fold interface and hence experience a major structural transition, display a characteristic sigmoidal curve. ii) An approximately linear change occurs for a amide protons being part of an unstructured segment, for which the major change is reflected in the decrease of solvent-coordination upon increasing the content of methanol, e.g. for residues 35 and 36 of Tyr7-PYY as well as from residues 6,7 11, 35 and 36 of PYY. The resonance frequencies of these protons shift to higher field with increasing methanol content. iii) Amide proton resonances of residues 20, 22, 25, 26, 29 and 30-32, all of which are pointing away from the back-folding interface, are shifted to lower field with increasing methanol content. These changes can be attributed to the fact that the isolated helix and hence the involved hydrogen bonds are stabilized in methanol.

Figure S4: ${ }^{3} \mathrm{~J}(\mathrm{HN} \alpha)$ scalar coupling constants for Tyr7-PYY in various water-methanol mixtures:

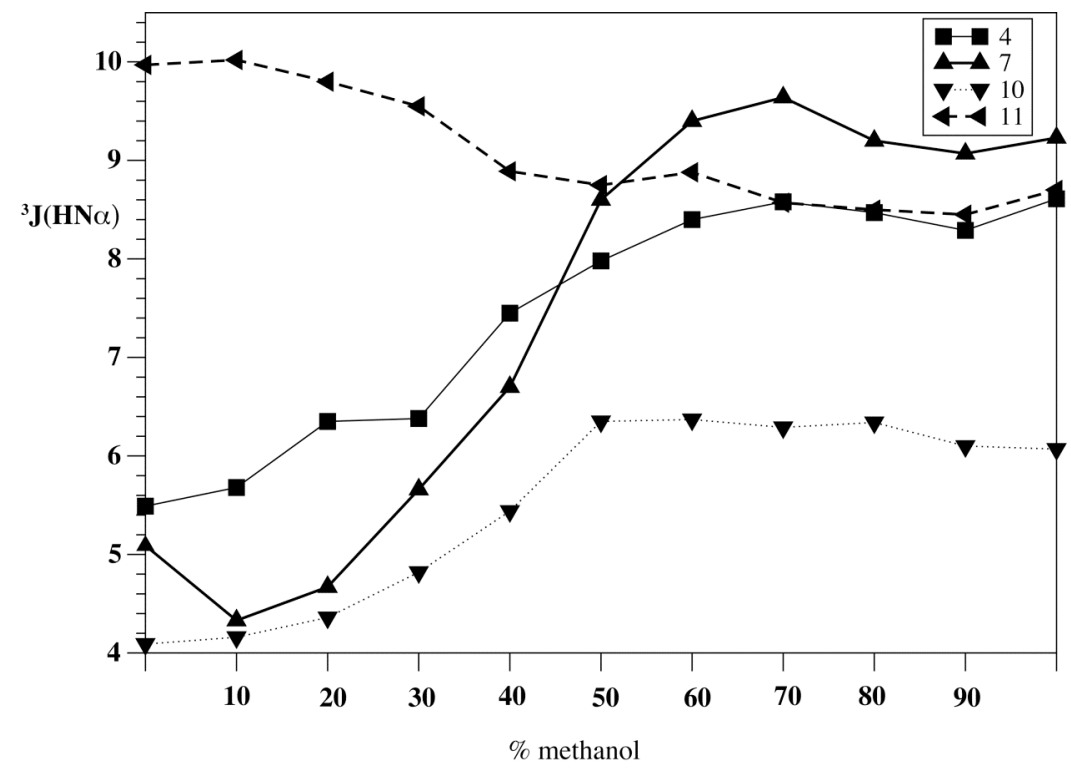


The data display sigmoidal curves. Interestingly, while the H-NOE data indicate that the N-terminal segment is largely flexible at $100 \%$ methanol, the scalar coupling data are only compatible with incomplete averaging of dihedral angles. While further work in our laboratory is in progress to investigate this issue in more detail, we speculate that the presence of Pro residues restricts conformational space for backbone rotameric states.

Figure S5: H-NOE data of PYY in various water-methanol mixtures (percentage methanol indicated).

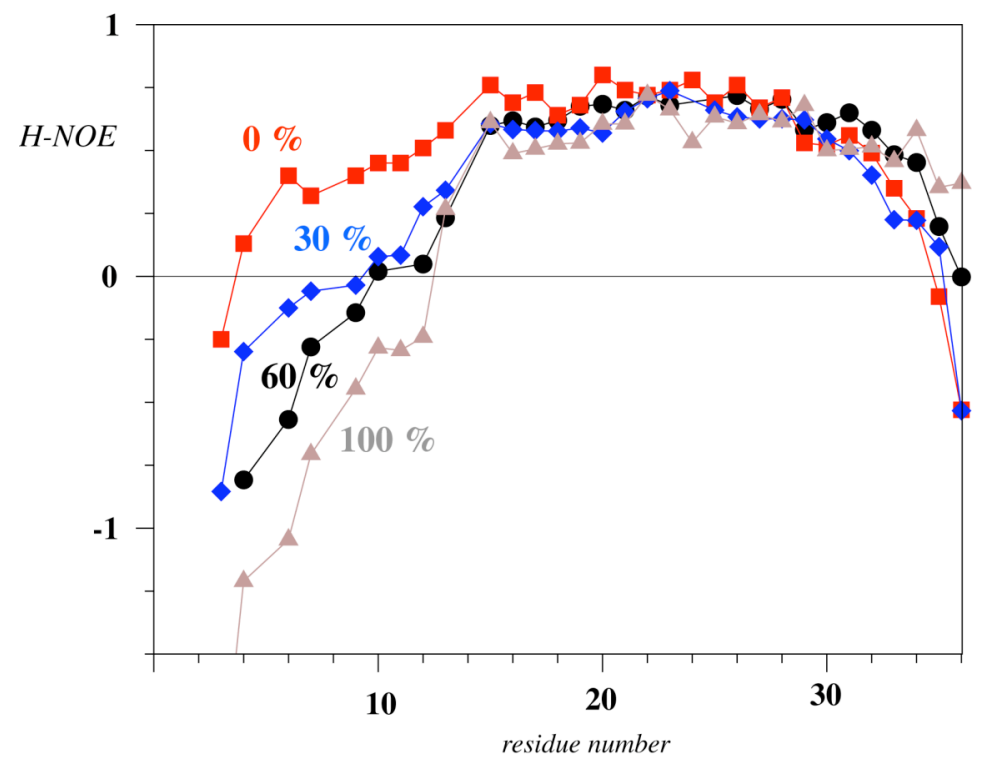

\section{Dimerization of PYY and Tyr7-PYY}

It should be noted that the related avian polypeptide (aPP) exists in dimeric form in the crystal structure. Therein, the interface between the monomeric subunits is formed by a hydrophobic cluster, in which the $\pi$-systems of Tyr7, Tyr21 and Phe 20 stack onto each other. In PYY, position 7 is occupied by Ala, and hence we decided to replace it by Tyr. The solution structure of Tyr7-PYY and the ${ }^{15} \mathrm{~N}\left\{{ }^{1} \mathrm{H}\right\}-$ NOE data indicate that the backbone of Tyr7-PYY is very similar to PYY, and slightly less rigid in the turn region comprising residues 10 to 14 . The $\mathrm{K}_{\mathrm{d}}$ for homodimerization of PYY is reported to be $22 \mathrm{mM}$ at $\mathrm{pH}$ 5. It is difficult to measure $\mathrm{K}_{\mathrm{d}}$ 's in that range due to the limited solubility of the peptide at very high concentrations, but dilution NMR experiments conducted with PYY suggest a similar value at $\mathrm{pH}$ $4.1(35 \pm 18 \mathrm{mM})$. It is also difficult to follow changes of backbone dynamics at very low concentrations to derive estimates of the contribution of dimerization to the stability of the back-fold. However, it is still possible to monitor chemical shift changes upon dilution. The amide proton frequency of Ala12 is very sensitive to the extent of back-folding: While it is at about $7.3 \mathrm{ppm}$ in PYY, it is measured to be 7.9 ppm in the strongly destabilized mutant Ala8. The amide proton shift of Ala12 shifts by -0.06 ppm upon dilution from $1 \mathrm{mM}$ to $20 \mathrm{uM}$. At the latter concentration the population of the monomer is higher than $99 \%$, while it is between $91 \%$ and $95 \%$ at $1 \mathrm{mM}$ for $\mathrm{K}_{\mathrm{d}}$ 's in the range between $20-40 \mathrm{mM}$. The data indicate that the dimer contributes to the stability of the helical hairpin only to a small extent, so that the gross effects must be due to changes within the monomer. We therefore feel justified to treat the system as a monomer to simplify the analysis.

\section{Turn geometry optimizations and energy calculations}

The turn segments of aPP and PYY comprising residues 11 - 15 were geometry optimized both in fully extended and in their native conformations. For the optimization of the native turn conformation, the coordinates of the backbone heavy atoms of residues 11 and 15 were held fixed in the positions as initially derived from the crystal structure of aPP (1) and the NMR structure of PYY (2), respectively. Geometry optimizations were performed using the B3LYP method (3) and the lacvp 
double- $\zeta$ quality basis set, as implemented in the Jaguar 4.2 program (4). Polarization effects from the surrounding solvent were taken into account by using a dielectric cavity model with a dielectric constant of $\varepsilon=78.8$ and a probe radius of 1.4. Table S2 summarizes the electronic energies after optimization of aPP and PYY in extended and turn conformation and the energy difference associated with the conformational change from extended to turn structures.

Table S1

\begin{tabular}{|c|c|c|c|}
\hline $\begin{array}{c}\text { Molecule } \\
\text { AA seq 11-15 }\end{array}$ & conformation & el. energy [Hartree] & $\begin{array}{c}\Delta \mathbf{E}_{\text {ext }>\text { turn }}[\mathrm{kcal} / \mathbf{m o l e}] \\
\text { (error is on the order of 5 kcal/mole) }\end{array}$ \\
\hline \hline aPP & extended & $-1922,551967$ & $-5,70$ \\
DAPYE & turn & $-1922,561057$ & 7,63 \\
\hline PYY & extended & $-1919,149773$ & \\
\hline DASPE & turn & $-1919,137608$ & \\
\hline
\end{tabular}

Figure S6: Optimized turn conformation of PYY. Atoms held fixed during the optimization are labeled with a star.

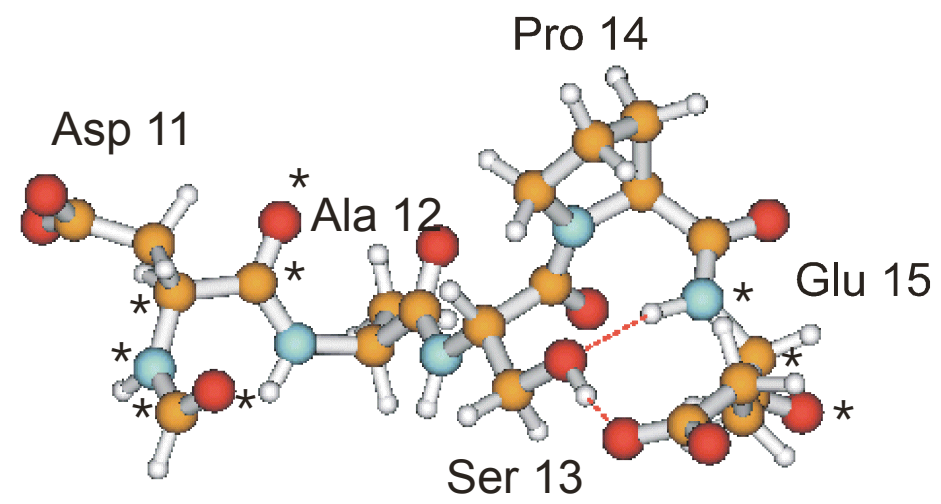

Turn formation in aPP is predicted to be slightly favourable, by contrast to turn formation in PYY, for which calculations predict a slightly unfavourable energy. As mentioned in the manuscript text, turn formation in PYY is not consistent with a Pro in position 13 (by contrast to aPP), nor does it tolerate substitution of Ser13 by Ala. Together, these observations point towards a critical role played by Ser13 in turn formation of PYY, rather than a geometry constraining function exerted by Pro14. One possible mechanism as to how Ser13 may stabilize the turn is evident from Figure S4, showing two hydrogen bonds from and to the side-chain hydroxyl group of Ser13. One is between the hydrogen of the $\mathrm{OH}$ group and the side-chain carboxyl group of Glu15 $(\mathrm{d}=1.56 \AA)$, whilst the other involves the hydroxyl oxygen and the backbone amide group of Glu15 (d=1.91 $\AA$ ). Substitution of Ser13 by Ala may therefore remove two turn-supporting interactions and hence critically destabilize the back-folded structure.

\section{References:}

(1) Blundell, T. L., Pitts, J. E., Tickle, S. P. \& Wu, C. W. (1981). Proc. Natl. Acad. Sci. U S A 78, 4175-9.

(2) Lerch, M., Mayrhofer, M. \& Zerbe, O. (2004). J. Mol. Biol. 339, 1153-68.

(3) Becke, A. D. (1993) J. Chem. Phys. 98, 5648-52.

(4) Schrödinger, Inc., Portland, Oregon, Jaguar 4.2, 2000. 
Figure S7: CD spectra of pPYY mutants ( $\alpha$-hairpin turn region), pPYY and NPY. Spectra were recorded in $1 \mathrm{mM}$ Na-acetate buffer $(\mathrm{pH} 4.4)$ at $298 \mathrm{~K}$.

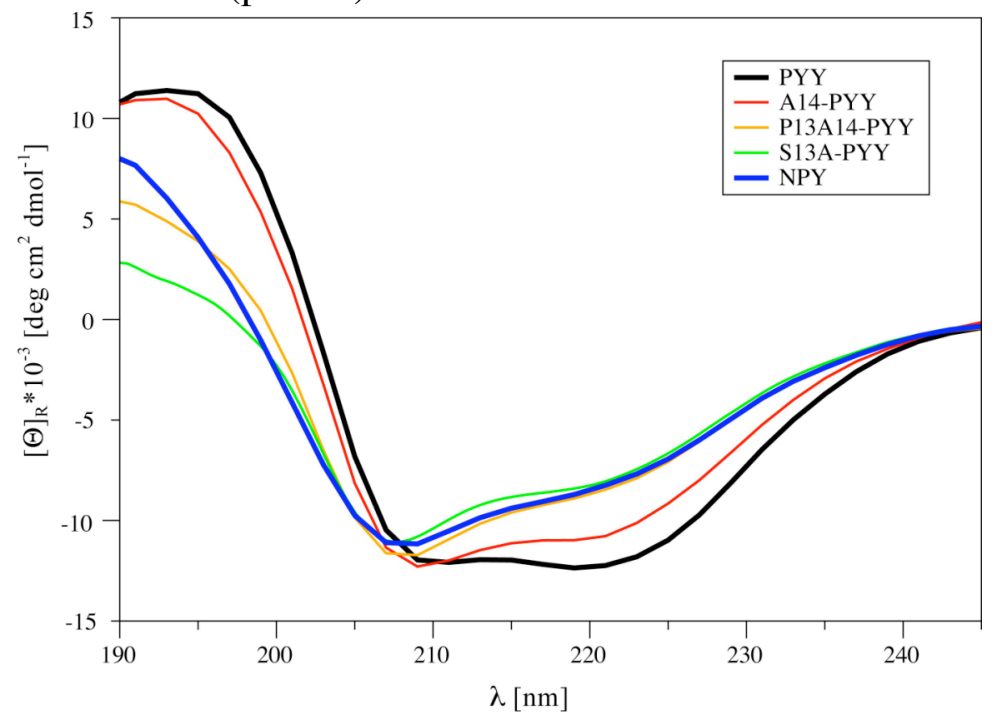

Figure S8: CD spectra of pPYY Pro->Ala mutants (N-terminal segment), pPYY and NPY. Spectra were recorded in $1 \mathrm{mM}$ Na-acetate buffer $(\mathrm{pH} 4.4)$ at $298 \mathrm{~K}$.

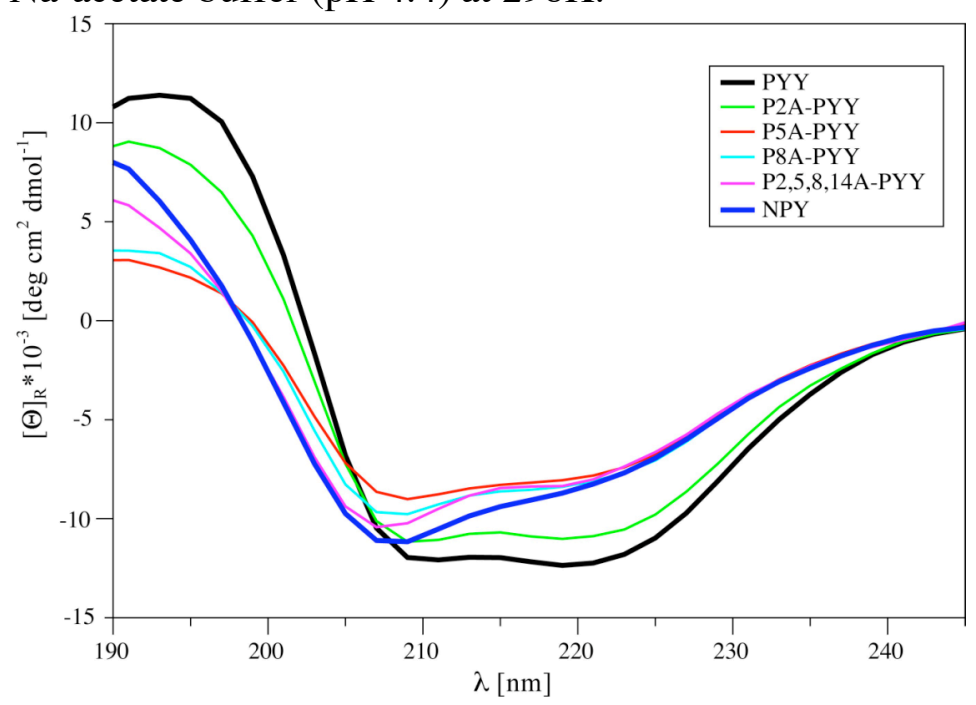

Figure S9: CD spectra of pPYY, Y7-pPYY, pPYY single Tyr->Ala mutants and NPY. Spectra were recorded in $1 \mathrm{mM}$ Na-acetate buffer $(\mathrm{pH} 4.4)$ at $298 \mathrm{~K}$.

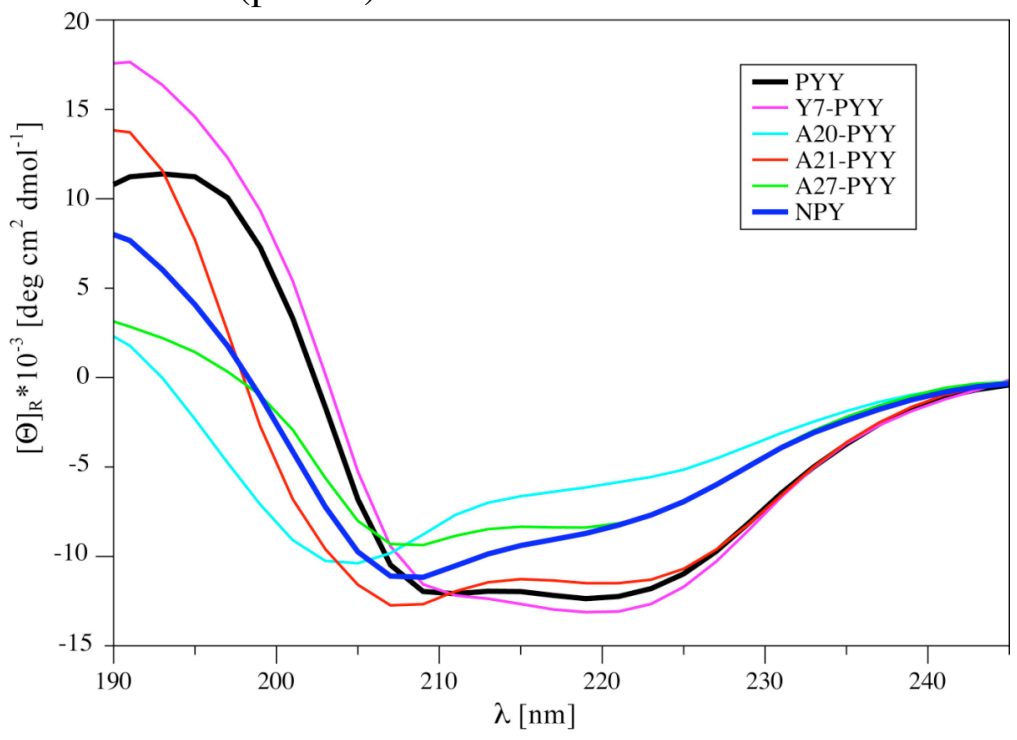


Table S2: Chemical shifts for 2mM P14A-pPYY in $90 \% \mathrm{H}_{2} \mathrm{O} / 10 \%{ }^{2} \mathrm{H}_{2} \mathrm{O}$, pH 4.1, T 301K, referenced to the signal of residual $\mathrm{HDO}$ at $4.72 \mathrm{ppm}$.

\begin{tabular}{|c|c|c|c|c|c|}
\hline $\mathrm{N}$ & Res. & $\mathrm{H}^{\mathrm{N}}$ & $\mathrm{H}^{\prime \alpha}$ & $\mathrm{H}^{\beta}$ & others \\
\hline 1 & TYR & - & 4.43 & $2.97,3.17$ & $\delta \mathrm{H} 7.18,7.18 ; \varepsilon \mathrm{H} \mathrm{6.84,6.84; \eta \textrm {OH } -}$ \\
\hline 2 & PRO & & 4.47 & $1.90,2.29$ & $\gamma \mathrm{CH}_{2} 1.91,1.91 ; \delta \mathrm{CH}_{2} 3.21,3.65$ \\
\hline 3 & ALA & 8.38 & 4.32 & 1.33 & \\
\hline 4 & LYS & 8.26 & 3.68 & $1.40,1.57$ & $\gamma \mathrm{CH}_{2} 1.08,1.08 ; \delta \mathrm{CH}_{2} 1.25,1.25 ; \varepsilon \mathrm{CH}_{2} 2.91,2.91 ; \xi \mathrm{NH}_{3}^{+}-$ \\
\hline 5 & PRO & & 4.34 & $1.85,2.27$ & $\gamma \mathrm{CH}_{2} 1.77,1.77 ; \delta \mathrm{CH}_{2} 3.19,3.50$ \\
\hline 6 & GLU & 8.45 & 4.22 & $1.85,1.93$ & $\gamma \mathrm{CH}_{2} 2.36,2.36 ; \varepsilon \mathrm{H}-$ \\
\hline 7 & ALA & 8.31 & 3.69 & 1.11 & \\
\hline 8 & PRO & & 4.31 & $1.77,2.08$ & $\gamma \mathrm{CH}_{2} 1.61,1.61 ; \delta \mathrm{CH}_{2} 2.93,2.93-$ \\
\hline 9 & GLY & 8.22 & $3.85,3.97$ & & \\
\hline 10 & GLU & 8.34 & 4.09 & $1.98,1.98$ & $\gamma \mathrm{CH}_{2} 2.37,2.37 ; \varepsilon \mathrm{H}-$ \\
\hline 11 & ASP & 8.51 & 4.62 & $2.74,2.79$ & $\delta \mathrm{H}-$ \\
\hline 12 & ALA & 7.46 & 4.20 & 1.32 & \\
\hline 13 & SER & 8.35 & 4.40 & $4.01,4.30$ & $\gamma \mathrm{OH}-$ \\
\hline 14 & ALA & 8.76 & 4.09 & 1.46 & \\
\hline 15 & GLU & 8.49 & 4.11 & $1.97,2.08$ & $\gamma \mathrm{CH}_{2} 2.39,2.39 ; \varepsilon \mathrm{H}-$ \\
\hline 16 & GLU & 7.87 & 3.95 & $1.97,2.23$ & $\gamma \mathrm{CH}_{2} 2.32,2.39 ; \varepsilon \mathrm{H} \mathrm{-}$ \\
\hline 17 & LEU & 8.40 & 3.97 & $1.56,1.82$ & $\gamma \mathrm{H} 1.32 ; \delta \mathrm{CH}_{3} 0.81,0.81$ \\
\hline 18 & SER & 8.28 & 4.26 & $4.02,4.02$ & $\gamma \mathrm{OH}-$ \\
\hline 19 & ARG & 7.95 & 4.11 & $1.81,1.91$ & $\gamma \mathrm{CH}_{2} 1.60,1.60 ; \delta \mathrm{CH}_{2} 3.18,3.18 ; \varepsilon \mathrm{NH} 7.34 ; \eta \mathrm{NH}_{2}-,-$ \\
\hline 20 & TYR & 8.05 & 4.33 & $2.95,3.12$ & 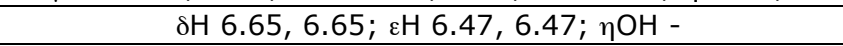 \\
\hline 21 & TYR & 8.64 & 3.96 & $2.94,3.07$ & $\delta \mathrm{H} \mathrm{7.05,7.05; \varepsilon H} \mathrm{6.80,6.80;} \eta \mathrm{OH}-$ \\
\hline 22 & ALA & 7.98 & 4.11 & 1.51 & \\
\hline 23 & SER & 8.17 & 4.29 & $3.93,4.03$ & $\gamma \mathrm{OH}-$ \\
\hline 24 & LEU & 8.60 & 4.00 & $1.60,1.60$ & $\gamma \mathrm{H} 1.17 ; \delta \mathrm{CH}_{3} 0.82,0.82$ \\
\hline 25 & ARG & 8.08 & 3.92 & $1.86,1.86$ & $\gamma \mathrm{CH}_{2} 1.55,1.71 ; \delta \mathrm{CH}_{2} 3.14,3.20 ; \varepsilon \mathrm{NH} 7.24 ; \eta \mathrm{NH}_{2}-,-$ \\
\hline 26 & HIS & 7.87 & 4.41 & $3.29,3.36$ & $\delta^{1} \mathrm{NH}-; \delta^{2} \mathrm{H} 7.27 ; \varepsilon^{1} \mathrm{H} 8.62 ; \varepsilon^{2} \mathrm{NH}-$ \\
\hline 27 & TYR & 8.18 & 4.09 & $3.14,3.14$ & $\delta \mathrm{H} 6.96,6.96 ; \varepsilon \mathrm{H} \mathrm{6.69,6.69;} \mathrm{\eta OH} \mathrm{-}$ \\
\hline 28 & LEU & 8.55 & 3.86 & $1.77,1.77$ & 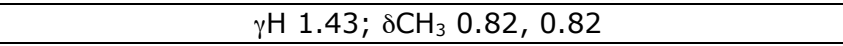 \\
\hline 29 & ASN & 7.96 & 4.41 & $2.78,2.83$ & $\delta \mathrm{NH}_{2} 6.86,7.55$ \\
\hline 30 & LEU & 7.66 & 4.07 & $1.40,1.64$ & $\gamma \mathrm{H} 1.48 ; \delta \mathrm{CH}_{3} 0.73,0.73$ \\
\hline 31 & VAL & 7.84 & 3.82 & 1.92 & $\gamma \mathrm{CH}_{3} 0.62,0.62$ \\
\hline 32 & THR & 7.74 & 4.23 & 4.15 & $\gamma \mathrm{CH}_{3} 1.18 ; \gamma \mathrm{OH}-$ \\
\hline 33 & ARG & 7.84 & 4.20 & $1.82,1.82$ & $\gamma \mathrm{CH}_{2} 1.62,1.62 ; \delta \mathrm{CH}_{2} 3.13,3.13 ; \varepsilon \mathrm{NH} 7.15 ; \eta \mathrm{NH}_{2-,-}$ \\
\hline 34 & GLN & 8.07 & 4.16 & $1.86,1.95$ & $\gamma \mathrm{CH}_{2} 2.28,2.28 ; \varepsilon \mathrm{NH}_{2}-,-$ \\
\hline 35 & ARG & 8.10 & 4.15 & $1.63,1.63$ & $\gamma \mathrm{CH}_{2} 1.36,1.44 ; \delta \mathrm{CH}_{2} 3.06,3.06 ; \varepsilon \mathrm{NH} 7.09 ; \eta \mathrm{NH}_{2}-,-$ \\
\hline 36 & TYR & 8.01 & 4.52 & $2.85,3.07$ & 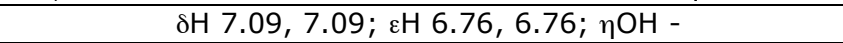 \\
\hline
\end{tabular}


Figure S10: Sequence plot of restraints used during the structure calculation of P14A-pPYY.

10

30

Y P A K P E P G D A S E E L S R Y A S L R Y L N L V T R R Y

$d_{\mathrm{NN}}(i, i+1)$

$d_{\alpha \mathrm{N}}(i, i+1)$

$d_{\beta \mathrm{N}}(i, i+1)$

$d_{\mathrm{NN}}(i, i+2)$

$d_{\alpha \mathrm{N}}(i, i+2)$

$d_{\alpha \mathrm{N}}(i, i+3)$

$d_{\alpha \beta}(i, i+3)$

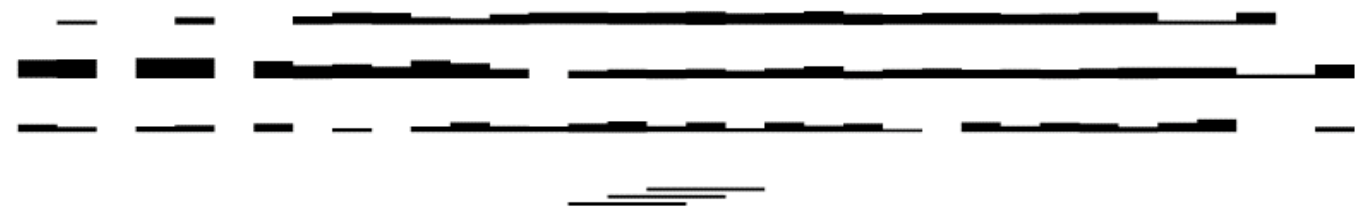

$d_{\alpha \mathrm{N}}(i, i+4)$

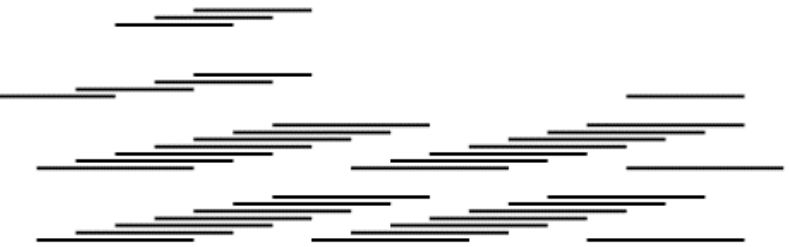

Table S3: Information on the structure calculation of P14A-pPYY in solution.

\begin{tabular}{|c|c|c|}
\hline \multirow[t]{2}{*}{ Distance restraints } & Total & 342 \\
\hline & Intra-residual & 84 \\
\hline & Sequential $(i-j=1)$ & 140 \\
\hline & $\operatorname{Medium}(i-j=2,3,4)$ & 99 \\
\hline & Long-range & 19 \\
\hline Dihedral angle restraints & & 147 \\
\hline \multicolumn{3}{|l|}{$\operatorname{RMSD}(\AA)^{\mathrm{a}}$} \\
\hline & Pro5-Val31 all heavy atoms & $2.48 \pm 0.80$ \\
\hline & Leu14-Arg29 backbone & $0.59 \pm 0.30$ \\
\hline & Leu14-Arg29 all heavy atoms & $1.46 \pm 0.29$ \\
\hline \multicolumn{3}{|l|}{ Structure check ${ }^{\mathrm{b}}$ (Average \%) } \\
\hline & Tyr1-Tyr36 & $98.8(1.2)$ \\
\hline \multirow[t]{2}{*}{ NOE constraint violations } & Number $>0.1 \AA$ & 1 \\
\hline & $\operatorname{Maximum}(\AA)$ & 0.21 \\
\hline Dihedral angle constraint violations & Number $>2.5$ degrees & 0 \\
\hline \multirow[t]{3}{*}{ AMBER energies $(\mathrm{kcal} / \mathrm{mol})^{\mathrm{c}}$} & Total & -581.8 \\
\hline & Van der Waals & -31.8 \\
\hline & Electrostatic & -875.1 \\
\hline \multicolumn{3}{|l|}{${ }^{\mathrm{a}} \mathrm{N}, \mathrm{C}_{\alpha}, \mathrm{C}^{\prime}$ atoms. } \\
\hline \multicolumn{3}{|c|}{$\begin{array}{l}\text { Percentage of the } \phi, \varphi \text { angles falling into the most favored (disallowed) regions within the allowed } \\
\text { Ramachandran regions for the } 20 \text { refined structures. }\end{array}$} \\
\hline
\end{tabular}


Table S4: Chemical shifts for $1 \mathrm{mM}$ A7Y-pPYY in $90 \% \mathrm{H}_{2} \mathrm{O} / 10 \%{ }^{2} \mathrm{H}_{2} \mathrm{O}$, pH 4.1, $\mathrm{T} 301 \mathrm{~K}$, referenced to the signal of residual HDO at $4.72 \mathrm{ppm}$.

\begin{tabular}{|c|c|c|c|c|c|}
\hline $\mathrm{N}$ & Res. & $\mathrm{H}^{\mathrm{N}}$ & $\mathrm{H}^{\mathrm{a}}$ & $\mathrm{H}^{\mathrm{b}}$ & others \\
\hline 1 & TYR & - & 4.46 & $3.01,3.19$ & $8 \mathrm{H} \mathrm{7.22,} \mathrm{7.22;} \varepsilon \mathrm{H} \mathrm{6.89,6.89;} \eta \mathrm{OH}-$ \\
\hline 2 & PRO & & 4.51 & $1.98,2.32$ & $\gamma \mathrm{CH}_{2} 1.91,1.91 ; \delta \mathrm{CH}_{2} 3.20,3.67$ \\
\hline 3 & ALA & 8.38 & 4.37 & 1.35 & \\
\hline 4 & LYS & 8.27 & 3.48 & $1.35,1.52$ & $\gamma \mathrm{CH}_{2} 0.94,1.22 ; \delta \mathrm{CH}_{2} 1.59,1.59 ; \varepsilon \mathrm{CH}_{2} 2.93,2.93 ; \xi \mathrm{NH}_{3}^{+}-$ \\
\hline 5 & PRO & & 4.39 & $1.88,2.29$ & $\gamma \mathrm{CH}_{2} 1.70,1.73 ; \delta \mathrm{CH}_{2} 3.08,3.45$ \\
\hline 6 & GLU & 8.54 & 4.34 & $1.87,1.91$ & $\gamma \mathrm{CH}_{2} 2.30,2.36 ; \varepsilon \mathrm{H} \mathrm{-}$ \\
\hline 7 & TYR & 8.6 & 3.7 & $2.62,2.72$ & $\delta \mathrm{H} 6.63,6.63 ; \varepsilon \mathrm{H} 6.61,6.61 ; \eta \mathrm{OH}-$ \\
\hline 8 & PRO & & 4.24 & $1.70,2.04$ & $\gamma \mathrm{CH}_{2} 1.26,1.51 ; \delta \mathrm{CH}_{2} 2.37,3.13$ \\
\hline 9 & GLY & 5.45 & $3.56,4.25$ & & \\
\hline 10 & GLU & 8.44 & 4.17 & $2.06,2.06$ & $\gamma \mathrm{CH}_{2} 2.48,2.48 ; \varepsilon \mathrm{H}-$ \\
\hline 11 & ASP & 8.41 & 4.73 & $2.72,2.87$ & $\delta \mathrm{H}-$ \\
\hline 12 & ALA & 7.09 & 4.16 & 1.16 & \\
\hline 13 & SER & 8.32 & 4.68 & $4.02,4.39$ & $\gamma \mathrm{OH}-$ \\
\hline 14 & PRO & & 4.28 & $1.98,2.41$ & $\gamma \mathrm{CH}_{2} 2.08,2.22 ; \delta \mathrm{CH}_{2} 3.91,3.96$ \\
\hline 15 & GLU & 8.51 & 4.15 & $1.97,2.11$ & $\gamma \mathrm{CH}_{2} 2.40,2.44 ; \varepsilon \mathrm{H}-$ \\
\hline 16 & GLU & 7.85 & 4.01 & $1.90,2.29$ & $\gamma \mathrm{CH}_{2} 2.36,2.41 ; \varepsilon \mathrm{H}-$ \\
\hline 17 & LEU & 8.51 & 4.02 & $1.55,1.64$ & $\gamma \mathrm{H} 1.87 ; \delta \mathrm{CH}_{3} 0.80,0.89$ \\
\hline 18 & SER & 8.48 & 4.36 & $4.05,4.05$ & $\gamma \mathrm{OH}-$ \\
\hline 19 & ARG & 7.88 & 4.16 & $1.95,1.95$ & $\gamma \mathrm{CH}_{2} 1.64,1.87 ; \delta \mathrm{CH}_{2} 3.18,3.25 ; \varepsilon \mathrm{NH} 7.35 ; \eta \mathrm{NH}_{2}-,-$ \\
\hline 20 & TYR & 8.01 & 4.30 & $3.08,3.08$ & $\delta \mathrm{H} 6.65,6.65 ; \varepsilon \mathrm{H} \mathrm{6.56,6.56;} \mathrm{\eta OH} \mathrm{-}$ \\
\hline 21 & TYR & 8.73 & 3.96 & $2.99,3.18$ & 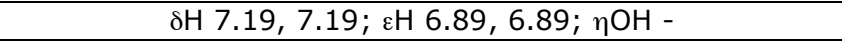 \\
\hline 22 & ALA & 7.98 & 4.16 & 1.55 & - \\
\hline 23 & SER & 8.24 & 4.32 & $3.93,4.05$ & $\gamma \mathrm{OH}-$ \\
\hline 24 & LEU & 8.88 & 3.99 & $1.03,1.71$ & $\gamma \mathrm{H} 1.54 ; \delta \mathrm{CH}_{3} 0.91,0.98$ \\
\hline 25 & ARG & 8.07 & 3.91 & $1.75,1.92$ & $\gamma \mathrm{CH}_{2} 1.59,1.59 ; \delta \mathrm{CH}_{2} 3.18,3.24 ; \varepsilon \mathrm{NH} 7.20 ; \eta \mathrm{NH}_{2}-,-$ \\
\hline 26 & HIS & 7.75 & 4.43 & $3.34,3.40$ & $\delta^{1} \mathrm{NH}-; \delta^{2} \mathrm{H} 7.37 ; \varepsilon^{1} \mathrm{H} 8.65 ; \varepsilon^{2} \mathrm{NH}-$ \\
\hline 27 & TYR & 8.24 & 4.09 & $3.20,3.20$ & $\delta \mathrm{H} 6.99,6.99 ; \varepsilon \mathrm{H} \mathrm{6.71,6.71;} \mathrm{\eta OH} \mathrm{-}$ \\
\hline 28 & LEU & 8.65 & 3.87 & $1.41,1.77$ & $\gamma \mathrm{H} \mathrm{1.88;} \delta \mathrm{CH}_{3} 0.83,0.90$ \\
\hline 29 & ASN & 7.91 & 4.44 & $2.80,2.87$ & $\delta \mathrm{NH}_{2} 6.86,7.52$ \\
\hline 30 & LEU & 7.64 & 4.11 & $1.41,1.67$ & $\gamma \mathrm{H} 1.52 ; \delta \mathrm{CH}_{3} 0.75,0.75$ \\
\hline 31 & VAL & 7.8 & 3.87 & 1.95 & $\gamma \mathrm{CH}_{3} 0.61,0.64$ \\
\hline 32 & THR & 7.72 & 4.20 & 4.24 & $\gamma \mathrm{CH}_{3} 1.22 ; \gamma \mathrm{OH}-$ \\
\hline 33 & ARG & 7.89 & 4.24 & $1.80,1.88$ & $\gamma \mathrm{CH}_{2} 1.64,1.64 ; \delta \mathrm{CH}_{2} 3.15,3.15 ; \varepsilon \mathrm{NH} 7.14 ; \eta \mathrm{NH}_{2}-,-$ \\
\hline 34 & GLN & 8.10 & 4.20 & $1.95,2.02$ & $\gamma \mathrm{CH}_{2} 2.31,2.31 ; \varepsilon \mathrm{NH}_{2}-,-$ \\
\hline 35 & ARG & 8.12 & 4.18 & $1.67,1.67$ & $\gamma \mathrm{CH}_{2} 1.40,1.47 ; \delta \mathrm{CH}_{2} 3.10,3.10 ; \varepsilon \mathrm{NH} 7.09 ; \eta \mathrm{NH}_{2}-,-$ \\
\hline 36 & TYR & 8.04 & 4.55 & $2.88,3.10$ & 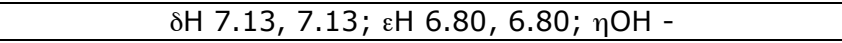 \\
\hline
\end{tabular}

Figure S11: Sequence plot for restraints used during the structure calculation of A7Y-PYY.

Y P A K P E P G E A S P E L S R Y Y A S L R Y L N L V T R R Y

$$
\begin{aligned}
& d_{\mathrm{NN}}(i, i+1) \\
& d_{\alpha \mathrm{N}}(i, i+1) \\
& d_{\beta \mathrm{N}}(i, i+1) \\
& d_{\mathrm{NN}}(i, i+2) \\
& d_{\alpha \mathrm{N}}(i, i+2) \\
& d_{\alpha \mathrm{N}}(i, i+3) \\
& d_{\alpha \beta}(i, i+3) \\
& d_{\alpha \mathrm{N}}(i, i+4)
\end{aligned}
$$
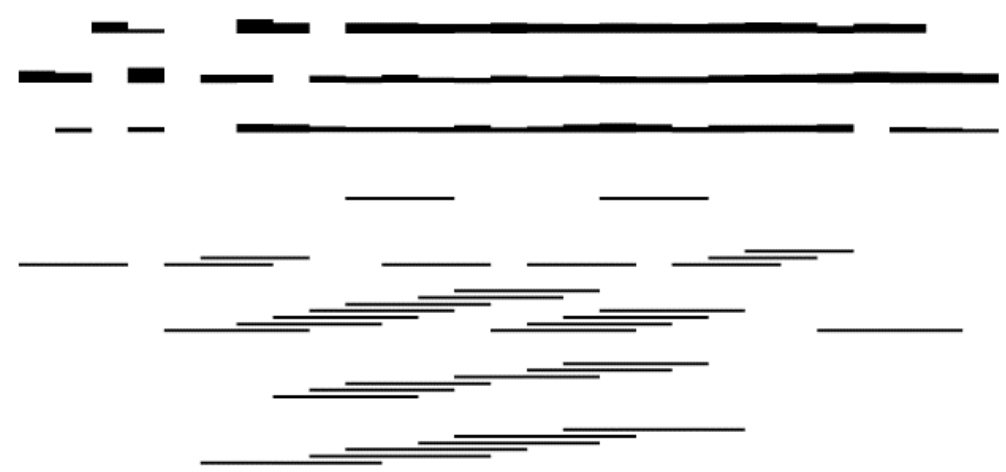
Table S5: Information on the structure calculation of A7Y-pPYY in solution.

\begin{tabular}{|c|c|c|}
\hline \multirow[t]{5}{*}{ Distance restraints } & Total & 552 \\
\hline & Intra-residual & 121 \\
\hline & Sequential $(i-j=1)$ & 161 \\
\hline & Medium $(i-j=2,3,4)$ & 152 \\
\hline & Long-range & 75 \\
\hline \multicolumn{3}{|l|}{ Dihedral angle restraints } \\
\hline \multirow{2}{*}{\multicolumn{3}{|c|}{$\operatorname{RMSD}(\AA)^{\mathrm{a}}$}} \\
\hline & & \\
\hline & Pro5-Val31 backbone & $1.29 \pm 0.44$ \\
\hline & Pro5-Val31 all heavy atoms & $2.21 \pm 0.48$ \\
\hline & Pro14-Val31 backbone & $0.81 \pm 0.30$ \\
\hline & Pro14-Val31 all heavy atoms & $1.72 \pm 0.37$ \\
\hline \multicolumn{3}{|l|}{ Structure check $^{\mathrm{b}}$ (Average \%) } \\
\hline & Tyr1-Tyr36 & $99.2(0.8)$ \\
\hline \multirow{2}{*}{ NOE constraint violations } & Number $>0.1 \AA$ & 0 \\
\hline & $\operatorname{Maximum}(\AA)$ & - \\
\hline $\begin{array}{l}\text { Dihedral angle constraint } \\
\text { violations }\end{array}$ & Number $>2.5$ degrees & 0 \\
\hline \multirow{3}{*}{ 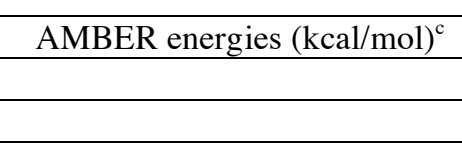 } & Total & -1112.0 \\
\hline & Van der Waals & 239.1 \\
\hline & Electrostatic & -2561.1 \\
\hline & & \\
\hline \multicolumn{3}{|l|}{${ }^{2} \mathrm{~N}, \mathrm{C}_{\alpha}, \mathrm{C}^{\prime}$ atoms. } \\
\hline \multicolumn{3}{|c|}{$\begin{array}{l}\text { Percentage of the } \phi, \varphi \text { angles falling into the most favored (disallowed) regions within the allowed } \\
\text { Ramachandran regions for the } 20 \text { refined structures. }\end{array}$} \\
\hline
\end{tabular}

Figure S12: Sequence plot of restraints used during the structure calculation of pPYY in $\mathrm{MeOH}$. 10 20 30 Y P A K P E P G E A S P E L S R Y Y S L R H L N L V T R Q R Y

$$
\begin{aligned}
& d_{\mathrm{NN}}(i, i+1) \\
& d_{\alpha \mathrm{N}}(i, i+1) \\
& d_{\beta \mathrm{N}}(i, i+1) \\
& d_{\mathrm{NN}}(i, i+2) \\
& d_{\alpha \mathrm{N}}(i, i+2) \\
& d_{\alpha \mathrm{N}}(i, i+3) \\
& d_{\alpha \beta}(i, i+3) \\
& d_{\alpha \mathrm{N}}(i, i+4)
\end{aligned}
$$

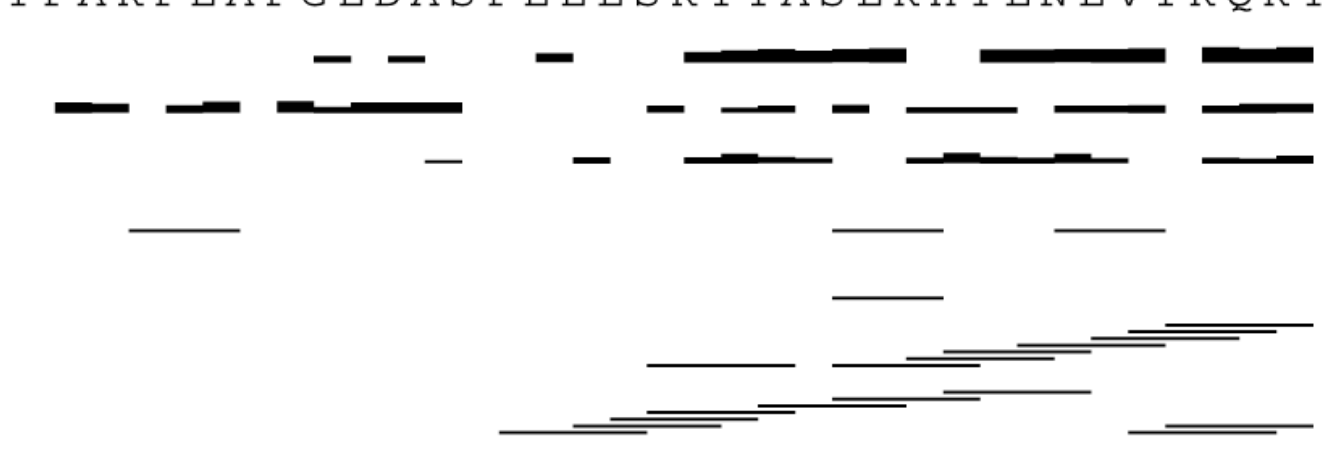


Table S6: Chemical Shifts of pPYY, $1 \mathrm{mM}, \mathrm{d}^{3}-\mathrm{MeOH}, 301 \mathrm{~K}$, referenced to residual $\mathrm{CD}_{2} \mathbf{H O H}$ at 3.31 ppm.

\begin{tabular}{|c|c|c|c|c|c|}
\hline $\mathrm{N}$ & Res. & $\mathrm{H}^{\mathrm{N}}$ & $\mathrm{H}^{\mathrm{a}}$ & $\mathrm{H}^{\mathrm{b}}$ & others \\
\hline 1 & Tyr & - & - &,-- & $\delta \mathrm{H}-,-; \varepsilon \mathrm{H}-,-; \eta \mathrm{OH}-$ \\
\hline 2 & Pro & & 4.43 & $1.97,2.22$ & $\gamma \mathrm{CH}_{2-,-} ; \delta \mathrm{CH}_{2}-,-$ \\
\hline 3 & Ala & 8.29 & 4.32 & 1.38 & \\
\hline 4 & Lys & 8.16 & 4.63 & $1.86,1.86$ & $\gamma \mathrm{CH}_{2} 1.69,1.69 ; \delta \mathrm{CH}_{2} 1.51,1.51 ; \varepsilon \mathrm{CH}_{2}-,-; \xi \mathrm{NH}_{3}^{+}-$ \\
\hline 5 & Pro & & 4.49 &,-- & $\gamma \mathrm{CH}_{2} 2.09,2.09 ; \delta \mathrm{CH}_{2} 3.65,3.80$ \\
\hline 6 & Glu & 8.29 & 4.36 & $1.94,1.94$ & $\gamma \mathrm{CH}_{2} 2.11,2.43 ; \varepsilon \mathrm{H} \mathrm{-}$ \\
\hline 7 & Ala & 8.07 & 4.63 & 1.34 & \\
\hline 8 & Pro & & 4.35 & $1.97,2.23$ & $\gamma \mathrm{CH}_{2-,-} ; \delta \mathrm{CH}_{2-,-}$ \\
\hline 9 & Gly & 8.52 & $3.80,3.92$ & & \\
\hline 10 & Glu & 8.07 & 4.29 & $2.42,2.42$ & $\gamma \mathrm{CH}_{2} 2.07,2.15 ; \varepsilon \mathrm{H}-$ \\
\hline 11 & Asp & 8.27 & 4.67 & $2.79,2.89$ & $\delta \mathrm{H}-$ \\
\hline 12 & Ala & 7.95 & 4.49 & 1.39 & \\
\hline 13 & Ser & 8.13 & 4.7 & $3.98,4.26$ & $\gamma \mathrm{OH}-$ \\
\hline 14 & Pro & & 4.3 & $1.94,2.05$ & $\gamma \mathrm{CH}_{2} 1.69,1.69 ; \delta \mathrm{CH}_{2}-,-$ \\
\hline 15 & Glu & 8.24 & 4.09 & $2.21,2.21$ & $\gamma \mathrm{CH}_{2} 2.51,2.59 ; \varepsilon \mathrm{H}-$ \\
\hline 16 & Glu & 7.96 & 4.11 & $2.17,2.28$ & $\gamma \mathrm{CH}_{2} 2.51,2.51 ; \varepsilon \mathrm{H}-$ \\
\hline 17 & Leu & 8.34 & 4.11 & $1.86,1.86$ & $\gamma \mathrm{H} 1.50 ; \delta \mathrm{CH}_{3} 0.93,0.93$ \\
\hline 18 & Ser & 8.34 & 4.25 & $4.01,4.10$ & $\gamma \mathrm{OH}-$ \\
\hline 19 & Arg & 7.97 & 4.06 & $1.99,1.99$ & $\gamma \mathrm{CH}_{2} 1.67,1.67 ; \delta \mathrm{CH}_{2} 3.21,3.21 ; \varepsilon \mathrm{NH} 7.45 ; \eta \mathrm{NH}_{2--}-$ \\
\hline 20 & Tyr & 8.44 & 4.17 & $3.13,3.18$ & $\delta \mathrm{H} 6.76,6.76 ; \varepsilon \mathrm{H} \mathrm{6.56,6.56; \eta \textrm {OH } -}$ \\
\hline 21 & Tyr & 8.67 & 4.13 & $3.12,3.12$ & $\delta \mathrm{H} 7.16,7.16 ; \varepsilon \mathrm{H} 6.77,6.77 ; \eta \mathrm{OH}-$ \\
\hline 22 & Ala & 8.58 & 4.05 & 1.57 & \\
\hline 23 & Ser & 8.23 & 4.17 & $4.03,4.06$ & $\gamma \mathrm{OH}-$ \\
\hline 24 & Leu & 8.35 & 4.1 & $1.68,1.68$ & $\gamma \mathrm{H} \mathrm{1.50; \delta \textrm {CH } _ { 3 } 0 . 8 7 , 0 . 8 7}$ \\
\hline 25 & Arg & 8.49 & 3.96 & $1.99,1.99$ & $\gamma \mathrm{CH}_{2}-,-; \delta \mathrm{CH}_{2} 3.20,3.20 ; \varepsilon \mathrm{NH} 7.44 ; \eta \mathrm{NH}_{2}-,-$ \\
\hline 26 & His & 8.4 & 4.48 & $3.41,3.51$ & $\delta^{1} \mathrm{NH}-; \delta^{2} \mathrm{H} 7.30 ; \varepsilon^{1} \mathrm{H} 8.73 ; \varepsilon^{2} \mathrm{NH}-$ \\
\hline 27 & Tyr & 8.49 & 4.07 & $3.21,3.21$ & 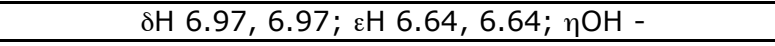 \\
\hline 28 & Leu & 8.7 & 3.92 & $1.97,1.97$ & 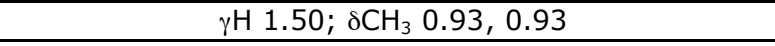 \\
\hline 29 & Asn & 8.55 & 4.38 & $2.75,3.10$ & $\delta \mathrm{NH}_{2} 7.02,7.79$ \\
\hline 30 & Leu & 8.01 & 3.97 & $1.74,1.74$ & $\gamma \mathrm{H} 1.60 ; \delta \mathrm{CH}_{3} 0.84,0.84$ \\
\hline 31 & Val & 8.27 & 3.65 & 1.98 & $\gamma \mathrm{CH}_{3} 0.75,0.84$ \\
\hline 32 & Thr & 8.03 & 3.87 & 4.36 & $\gamma \mathrm{CH}_{3} 1.28 ; \gamma \mathrm{OH}-$ \\
\hline 33 & Arg & 8.03 & 4.06 & $1.98,1.98$ & $\gamma \mathrm{CH}_{2} 1.86,1.86 ; \delta \mathrm{CH}_{2} 3.16,3.16 ; \varepsilon \mathrm{NH} 7.44 ; \eta \mathrm{NH}_{2}-,-$ \\
\hline 34 & $\mathrm{Gln}$ & 8.15 & 4.02 & $2.27,2.27$ & $\gamma \mathrm{CH}_{2} 2.07,2.52 ; \varepsilon \mathrm{NH}_{2}-,-$ \\
\hline 35 & Arg & 7.89 & 4.07 & $1.65,1.75$ & $\gamma \mathrm{CH}_{2} 1.43,1.52 ; \delta \mathrm{CH}_{2} 3.01,3.07 ; \varepsilon \mathrm{NH} 7.25 ; \eta \mathrm{NH}_{2}-,-$ \\
\hline 36 & Tyr & 7.77 & 4.52 & $2.84,3.20$ & $\delta \mathrm{H} 7.22,7.22 ; \varepsilon \mathrm{H} 6.67,6.67 ; \eta \mathrm{OH}-$ \\
\hline
\end{tabular}




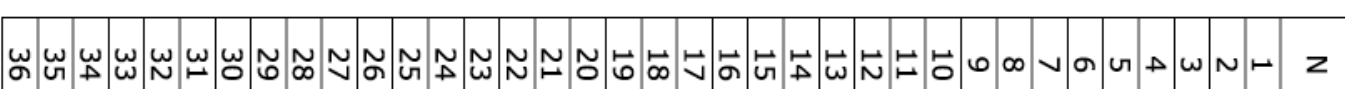

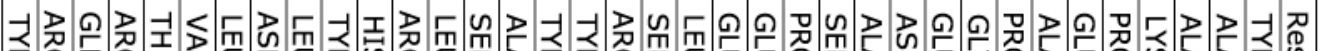

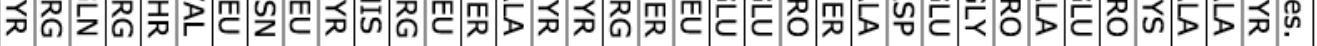

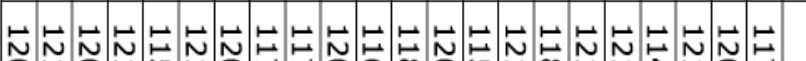

N N N N N

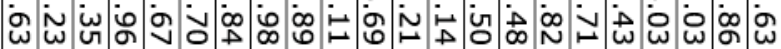

$\operatorname{s}: \vec{\sim}$

N|N

ân

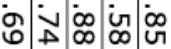

$\stackrel{\infty}{\infty}$

ติ जิ

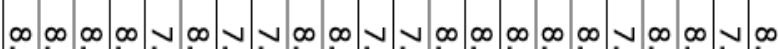

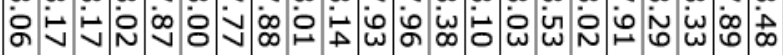

$\infty \vee \infty \infty$

$\infty \infty$

$\infty \infty \infty$

$\ddot{\omega}$ in

$\dot{\omega} \stackrel{\vec{\omega}}{\omega}$

o

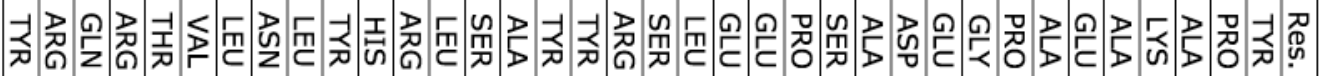

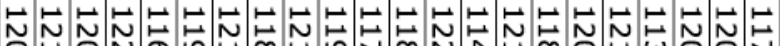

Non

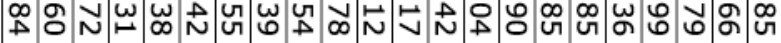

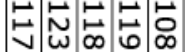

ㄴ $\omega$

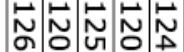

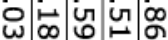

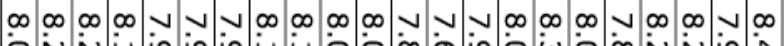

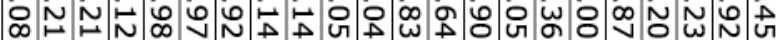

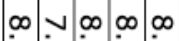

$\underset{N}{\infty}$ ने $N$

$\infty \infty \infty \infty \infty$

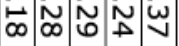

(D)

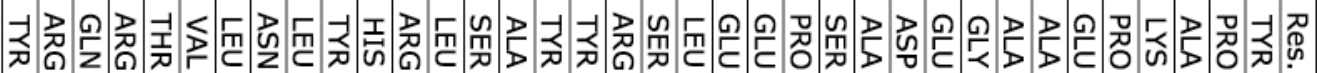

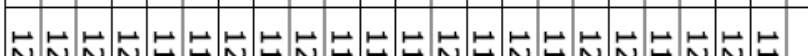

N) N

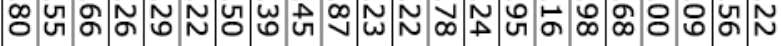

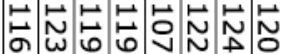

vi ư

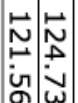

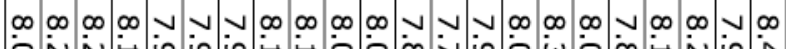

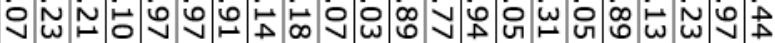

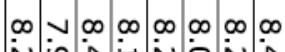

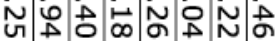

$\infty \infty$

$\stackrel{\sim}{\sim} \dot{\omega}$

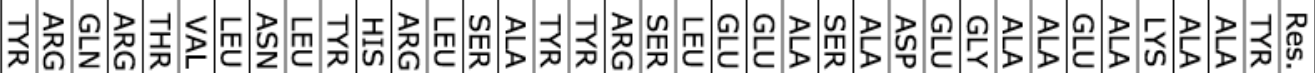

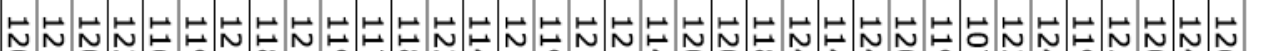

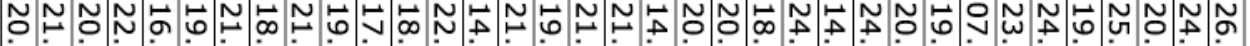

\&

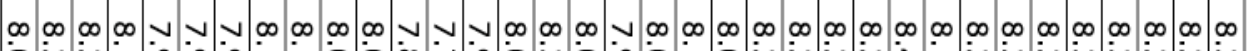

岀 N N $\tilde{N}_{0}$

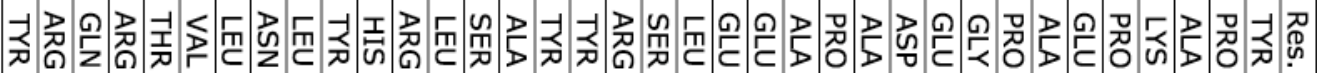

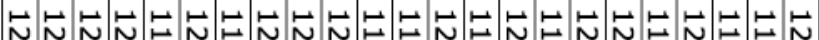

N N N N

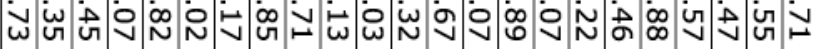

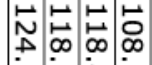

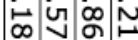

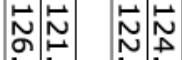

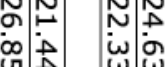

$2 \vec{\omega}$

(1)

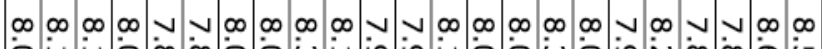

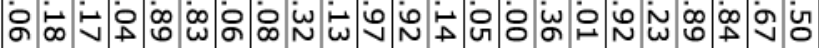

$\checkmark \infty \infty ⿻ \infty$

$v \vec{\infty} \underset{\omega}{\sim} \underset{\sim}{\omega}$

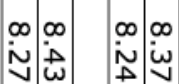

I

$\frac{1}{3}$

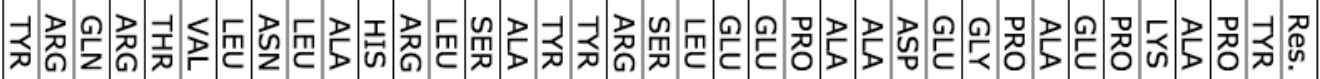

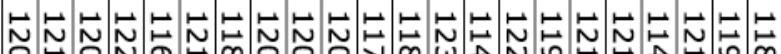

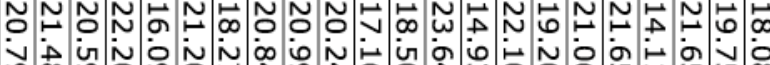

๖े

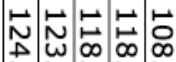

$\dot{0}$ कें

a

Nin

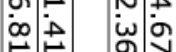

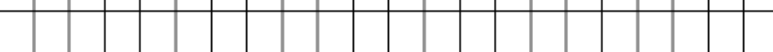

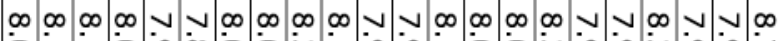

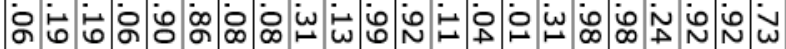

$\infty \sim \infty \infty \infty$

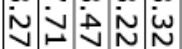

$\infty \infty$

$\stackrel{\infty}{\sim} \underset{\omega}{\omega}$

$\overrightarrow{0}$

.

. 
Table S8: Chemical shifts for pPYY Tyr->Ala mutants, $1 \mathrm{mM}$ samples in $90 \% \mathrm{H}_{2} \mathrm{O} / 10 \%{ }^{2} \mathrm{H}_{2} \mathrm{O}$, pH 4.1, $\mathrm{T} 301 \mathrm{~K}$, referenced to the signal of residual HDO at $4.72 \mathrm{ppm}$.

\begin{tabular}{|c|c|c|c|c|c|c|c|c|c|}
\hline \multirow{2}{*}{$\mathrm{N}$} & \multicolumn{3}{|c|}{ Y20A-pPYY } & \multicolumn{3}{|c|}{ Y21A-pPYY } & \multicolumn{3}{|c|}{ Y27A-pPYY } \\
\hline & Res. & $\mathrm{N}, \mathrm{ppm}$ & $\mathrm{H}^{\mathrm{N}}, \mathrm{ppm}$ & Res. & $\mathrm{N}, \mathrm{ppm}$ & $\mathrm{H}^{\mathrm{N}}, \mathrm{ppm}$ & Res. & $\mathrm{N}, \mathrm{ppm}$ & $\mathrm{H}^{\mathrm{N}}, \mathrm{ppm}$ \\
\hline 1 & TYR & & & TYR & & & TYR & & \\
\hline 2 & PRO & & & PRO & & & PRO & & \\
\hline 3 & ALA & 124.77 & 8.37 & ALA & 124.60 & 8.38 & ALA & 124.77 & 8.37 \\
\hline 4 & LYS & 121.49 & 8.24 & LYS & 122.91 & 8.26 & LYS & 121.34 & 8.23 \\
\hline 5 & PRO & & & PRO & & & PRO & & \\
\hline 6 & GLU & 120.85 & 8.42 & GLU & 122.01 & 8.46 & GLU & 120.99 & 8.41 \\
\hline 7 & ALA & 126.32 & 8.26 & ALA & 127.09 & 8.30 & ALA & 126.09 & 8.19 \\
\hline 8 & PRO & & & PRO & & & PRO & & \\
\hline 9 & GLY & 109.08 & 8.45 & GLY & 107.92 & 8.29 & GLY & 108.78 & 8.37 \\
\hline 10 & GLU & 119.61 & 8.13 & GLU & 118.70 & 8.31 & GLU & 119.17 & 8.22 \\
\hline 11 & ASP & 120.08 & 8.42 & ASP & 118.13 & 8.51 & ASP & 118.84 & 8.45 \\
\hline 12 & ALA & 123.86 & 8.05 & ALA & 122.75 & 7.53 & ALA & 123.35 & 7.78 \\
\hline 13 & SER & 116.56 & 8.24 & SER & 117.45 & 8.37 & SER & 117.00 & 8.30 \\
\hline 14 & PRO & & & PRO & & & PRO & & \\
\hline 15 & GLU & 118.59 & 8.41 & GLU & 117.76 & 8.46 & GLU & 117.97 & 8.45 \\
\hline 16 & GLU & 120.40 & 8.04 & GLU & 120.96 & 7.95 & GLU & 120.67 & 7.94 \\
\hline 17 & LEU & 121.17 & 8.13 & LEU & 120.87 & 8.35 & LEU & 121.09 & 8.24 \\
\hline 18 & SER & 114.52 & 8.08 & SER & 116.05 & 8.31 & SER & 114.00 & 8.21 \\
\hline 19 & ARG & 122.10 & 8.02 & ARG & 122.72 & 8.09 & ARG & 121.47 & 7.91 \\
\hline 20 & ALA & 122.94 & 8.05 & TYR & 120.58 & 8.15 & TYR & 121.11 & 8.01 \\
\hline 21 & TYR & 118.34 & 8.04 & ALA & 122.17 & 8.21 & TYR & 119.02 & 8.37 \\
\hline 22 & ALA & 122.63 & 8.04 & ALA & 120.12 & 7.89 & ALA & 121.91 & 8.06 \\
\hline 23 & SER & 113.40 & 8.02 & SER & 115.96 & 8.19 & SER & 114.14 & 7.90 \\
\hline 24 & LEU & 122.84 & 7.91 & LEU & 124.11 & 8.41 & LEU & 122.55 & 7.58 \\
\hline 25 & ARG & 118.91 & 8.01 & ARG & 118.51 & 7.84 & ARG & 118.36 & 7.79 \\
\hline 26 & HIS & 117.72 & 8.14 & HIS & 116.94 & 7.99 & HIS & 117.50 & 8.09 \\
\hline 27 & TYR & 120.13 & 8.10 & TYR & 120.28 & 8.14 & ALA & 123.45 & 8.10 \\
\hline 28 & LEU & 121.94 & 8.18 & LEU & 120.17 & 8.43 & LEU & 120.41 & 8.12 \\
\hline 29 & ASN & 118.62 & 8.19 & ASN & 118.07 & 8.02 & ASN & 118.70 & 8.25 \\
\hline 30 & LEU & 121.69 & 7.96 & LEU & 120.70 & 7.78 & LEU & 121.78 & 8.01 \\
\hline 31 & VAL & 119.54 & 8.00 & VAL & 117.52 & 7.87 & VAL & 120.31 & 8.01 \\
\hline 32 & THR & 116.45 & 8.00 & THR & 115.54 & 7.83 & THR & 117.26 & 8.08 \\
\hline 33 & ARG & 122.36 & 8.13 & ARG & 121.95 & 8.00 & ARG & 122.72 & 8.21 \\
\hline 34 & GLN & 120.76 & 8.23 & GLN & 120.31 & 8.15 & GLN & 121.11 & 8.28 \\
\hline 35 & ARG & 121.64 & 8.22 & ARG & 121.24 & 8.16 & ARG & 121.84 & 8.25 \\
\hline 36 & TYR & 120.88 & 8.08 & TYR & 120.65 & 8.05 & TYR & 120.99 & 8.10 \\
\hline
\end{tabular}


Table S9: Chemical shifts for pPYY Pro->Ala mutants $1 \mathrm{mM}$ samples in $90 \% \mathrm{H}_{2} \mathrm{O} / 10 \%{ }^{2} \mathrm{H}_{2} \mathrm{O}, \mathbf{3 0 0 m M}$ DPC, pH 6.0, T 310K, referenced to the signal of residual HDO at $4.63 \mathrm{ppm}$.

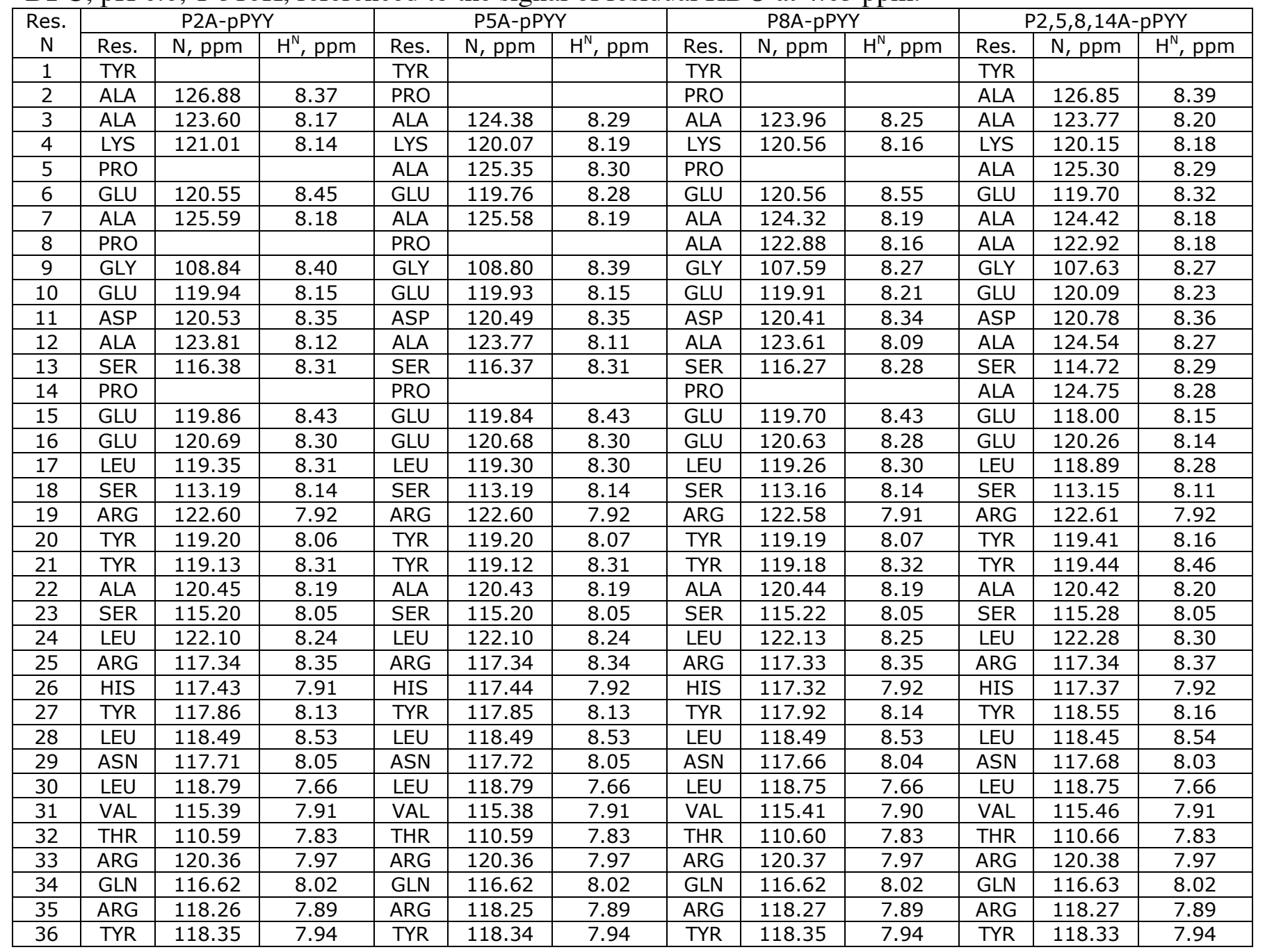




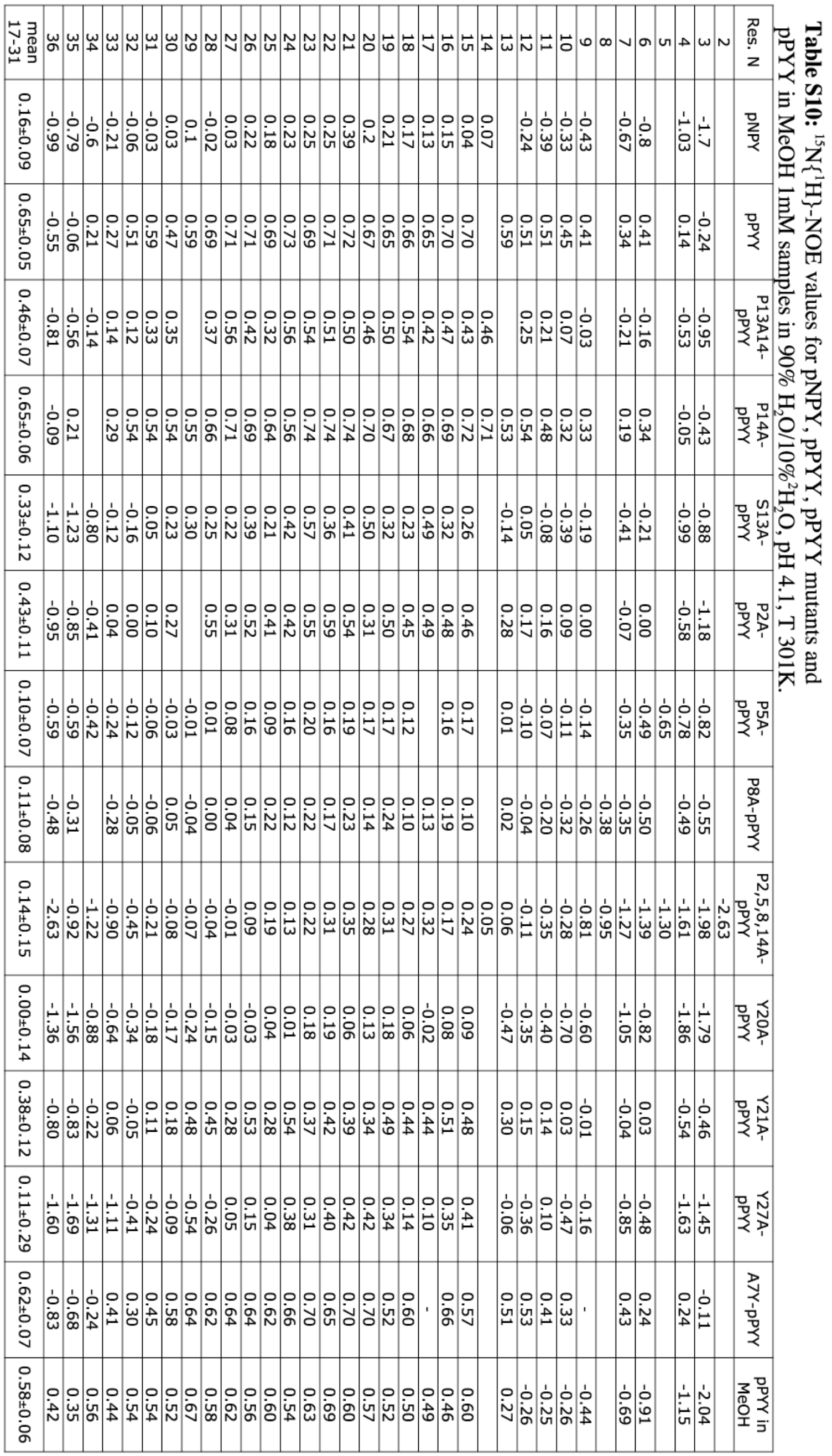


Table S11: ${ }^{15} \mathrm{~N}\left\{{ }^{1} \mathrm{H}\right\}-\mathrm{NOE}$ values for $\mathrm{pPYY}$ and pPYY Pro->Ala mutants $1 \mathrm{mM}$ samples in $90 \%$ $\mathrm{H}_{2} \mathrm{O} / 10 \%{ }^{2} \mathrm{H}_{2} \mathrm{O}, 300 \mathrm{mM}$ DPC, $\mathrm{pH}$ 6.0, T 310K.

\begin{tabular}{|c|c|c|c|c|c|}
\hline res. $\mathrm{N}$ & pPYY & P2A-pPYY & P5A-pPYY & P8A-pPYY & $\mathrm{P} 2,5,8,14 \mathrm{~A}-\mathrm{pPYY}$ \\
\hline 2 & & - & & & -2.31 \\
\hline 3 & -1.35 & -1.68 & -1.51 & -1.38 & -1.91 \\
\hline 4 & -1.30 & -1.44 & -1.45 & -1.35 & -1.45 \\
\hline 5 & & & -1.14 & & -1.12 \\
\hline 6 & -1.00 & -1.14 & -0.96 & -1.25 & -0.92 \\
\hline 7 & -0.92 & -1.09 & -1.04 & -1.16 & -1.21 \\
\hline 8 & & & & -1.18 & -1.13 \\
\hline 9 & -0.72 & -0.76 & -0.79 & -0.82 & -0.74 \\
\hline 10 & -0.64 & -0.65 & -0.69 & -0.60 & -0.41 \\
\hline 11 & -0.28 & -0.30 & -0.32 & -0.33 & -0.21 \\
\hline 12 & -0.41 & -0.51 & -0.49 & -0.45 & 0.06 \\
\hline 13 & 0.03 & 0.05 & -0.02 & -0.06 & 0.22 \\
\hline 14 & & & & & 0.37 \\
\hline 15 & 0.30 & 0.28 & 0.24 & 0.28 & 0.60 \\
\hline 16 & 0.45 & 0.38 & 0.38 & 0.36 & 0.32 \\
\hline 17 & 0.64 & 0.55 & 0.54 & 0.54 & 0.58 \\
\hline 18 & 0.59 & 0.58 & 0.54 & 0.57 & 0.59 \\
\hline 19 & 0.69 & 0.66 & 0.68 & 0.66 & 0.71 \\
\hline 20 & 0.63 & 0.63 & 0.63 & 0.61 & 0.54 \\
\hline 21 & 0.67 & 0.73 & 0.63 & 0.61 & 0.73 \\
\hline 22 & 0.68 & 0.65 & 0.60 & 0.66 & 0.58 \\
\hline 23 & 0.67 & 0.72 & 0.69 & 0.67 & 0.71 \\
\hline 24 & 0.73 & 0.73 & 0.70 & 0.67 & 0.74 \\
\hline 25 & 0.76 & 0.73 & 0.74 & 0.75 & 0.76 \\
\hline 26 & 0.77 & 0.73 & 0.75 & 0.72 & 0.73 \\
\hline 27 & 0.70 & 0.74 & 0.68 & 0.70 & 0.36 \\
\hline 28 & 0.71 & 0.68 & 0.67 & 0.71 & 0.74 \\
\hline 29 & 0.72 & 0.69 & 0.71 & 0.70 & 0.72 \\
\hline 30 & 0.67 & 0.62 & 0.64 & 0.64 & 0.68 \\
\hline 31 & 0.68 & 0.66 & 0.68 & 0.66 & 0.74 \\
\hline 32 & 0.58 & 0.57 & 0.62 & 0.60 & 0.58 \\
\hline 33 & 0.60 & 0.52 & 0.60 & 0.53 & 0.57 \\
\hline 34 & 0.51 & 0.49 & 0.50 & 0.52 & 0.47 \\
\hline 35 & 0.42 & 0.45 & 0.45 & 0.43 & 0.42 \\
\hline 36 & 0.32 & 0.33 & 0.34 & 0.32 & 0.33 \\
\hline $\begin{array}{l}\text { mean } \\
17-31\end{array}$ & $0.69 \pm 0.05$ & $0.67 \pm 0.06$ & $0.66 \pm 0.06$ & $0.66 \pm 0.06$ & $0.66 \pm 0.11$ \\
\hline
\end{tabular}


